\title{
NEOADYUVANCIA CON IMATINIB EN EL MANEJO DE GIST GIGANTE DEL TABIQUE RECTOVAGINAL*
}

\author{
Drs. Guillermo Bannura C. ${ }^{1}$, Cristián Gallardo M. ${ }^{1}$, Valeria Cornejo C. ${ }^{2}$ \\ 1 Servicio de Cirugía. \\ 2 Servicio de Anatomía Patológica. \\ Hospital Clínico San Borja Arriarán, Campus Centro, Facultad de Medicina, Universidad de Chile. \\ Santiago, Chile.
}

\begin{abstract}
Neo-adjuvant treatment with imatinib for a rectal gastrointestinal stromal tumor

Background: Rectal gastrointestinal stromal tumors (GIST) are rare. Neo-adjuvant therapy with imatinib is recommended for locally advanced or non-resectable tumors. Case report: We report a 63 years old woman with a malignant GIST located in the recto-vaginal septum which was initially considered nonresectable. The patient was treated with imatinib as induction therapy for three months. After this lapse the tumor was successfully excised using an endo-anal approach. Due to a tumor size over $5 \mathrm{~cm}$ and the presence of 13 mitoses per 50 high power fields, two bad prognostic factors, treatment with imatinib was maintained for 15 months after surgery. After 20 months of follow up, the patient is free of disease with complete fecal continence and with an adequate sexual life. Secondary effects of imatinib are gradually subsiding
\end{abstract}

Key words: GIST, imatinib, endo-anal excision.

\section{Resumen}

Introducción: Los tumores estromales gastrointestinales (GIST) del recto son muy raros. En la última década se ha planteado la neoadyuvancia con imatinib en los casos de tumores localmente avanzados o inicialmente irresecables. Caso clínico: Se presenta una paciente portadora de un GIST maligno del tabique rectovaginal inicialmente considerado irresecable que fue sometido a neoadyuvancia con Imatinib como terapia de inducción durante 3 meses, logrando una respuesta clínica notable que permitió realizar una resección local exitosa por vía endoanal. La presencia de factores pronósticos deletéreos (tamaño tumoral mayor de 5 $\mathrm{cm}$ y 13 mitosis por 50 campos de aumento mayor) determinó la mantención del tratamiento con Imatinib por 15 meses luego de la cirugía con el fin de reducir el riesgo de recidiva local. Luego de 20 meses de seguimiento la paciente está libre de enfermedad, con continencia fecal plena, ha recuperado la actividad sexual, aunque persisten algunos efectos residuales de la droga que están en franca disminución. Conclusión: La neoadyuvancia con imatinib se considera actualmente la terapia estándar en el manejo de los GIST localmente avanzados y/o irresecables.

Palabras clave: GIST rectal, imatinib, cirugía.

*Recibido el 6 de mayo de 2013 y aceptado para publicación el 9 de julio de 2013.

Los autores no refieren conflictos de interés.

Correspondencia: Dr. Guillermo Bannura C.

Las Limas 1622, Santiago, Chile.

gbannura@gtdmail.com 


\section{Introducción}

Los tumores estromales del tracto gastrointestinal (GIST) representan el 1\% de los tumores del tubo digestivo ${ }^{1}$. El compromiso del recto es poco frecuente, no superando en la mayoría de las series el $4 \%$ del total de los GIST $^{2}$. Aunque la cirugía resectiva es la principal terapia curativa en el manejo de estas lesiones, en la última década se ha empleado el Imatinib como terapia de inducción en pacientes con enfermedad localmente avanzada como una manera de reducir la morbilidad quirúrgica y/o permitir la resección en lesiones consideradas irresecables en la evaluación clínica inicial ${ }^{3-5}$. Se presenta un caso de GIST gigante localizado en el tabique rectovaginal sometido a neoadyuvancia con Imatinib seguido de resección local.

\section{Caso clínico}

Paciente de 63 años que consulta por dolor pelviano, asociado a baja de peso de $7 \mathrm{~kg}$ de 4 meses de evolución, disquecia y rectorragia ocasional. Al tacto rectal y vaginal se palpa una masa tumoral de gran tamaño, que compromete el tabique rectovaginal, fija a los planos profundos, de consistencia firme, que ulcera la mucosa rectal desde $1 \mathrm{~cm}$ por encima de la línea pectínea sin que sea posible precisar el límite proximal. La colonoscopia mostró un tumor ulcerado de recto inferior que ocupa el $50 \%$ del lumen. La tomografía computada (TC) de pelvis (Figuras 1-3) revela una masa de $10 \times 9 \mathrm{~cm}$ con una área central de necrosis que compromete la vagina, el cérvix y el recto, con realce periférico del medio de contraste endovenoso. La resonancia magnética (RM) de pelvis (Figura 4) muestra una gran masa sólida heterogénea $(9 \times 8 \times 6 \mathrm{~cm})$ que ocupa el fondo de saco rectouterino, infiltra la pared del recto, los parametrios y el cuello uterino, con adenopatías de la cavidad pelviana”. Biopsia: GIST con 5 mitosis en 10 campos de aumento mayor. IHQ: CD 117 y CD 34 intensamente positivo en las células tumorales. Se considera inicialmente irresecable, por lo que se decide neoadyuvancia con imatinib $400 \mathrm{mg}$ diarios, logrando una reducción del 50\% del tamaño tumoral al mes, con cierta movilidad al tacto. Luego de 2 meses de tratamiento en los cuales la paciente sube $4 \mathrm{~kg}$ de peso, se palpa una masa ovoidea blanda y móvil que compromete el tabique rectovaginal de $5 \mathrm{~cm}$ de diámetro mayor, límite superior definido, con depresión central al tacto rectal e indemnidad de la mucosa vaginal. A los 3 meses, la RM muestra una masa sólida de 5,5 × 3,2 × 2,5 cm, que respeta el plano anterior, dejando el cérvix y el útero libres (Figura 5). TC de tórax normal. Se interviene en posición de navaja sevillana. Infiltración de la mucosa vaginal con solución fisiológica. Resección local endoanal, reparando el tabique rectovaginal en 2 planos más plastía de los elevadores y cierre completo de la brecha rectal. Biopsia: tumor GIST de 4 x 3,5 x $3 \mathrm{~cm}$ con extensas áreas de hialinización estromal, presencia de células gigantes multinucleadas y 13 mitosis x 50 campos de aumento mayor (CAM). No se aprecia necrosis y la lesión compromete la submucosa, muscular propia y tejido adiposo perirrectal. CD 117 y CD 34 positivo intenso en el $100 \%$ de las células neoplásicas. Actina músculo liso (-) y

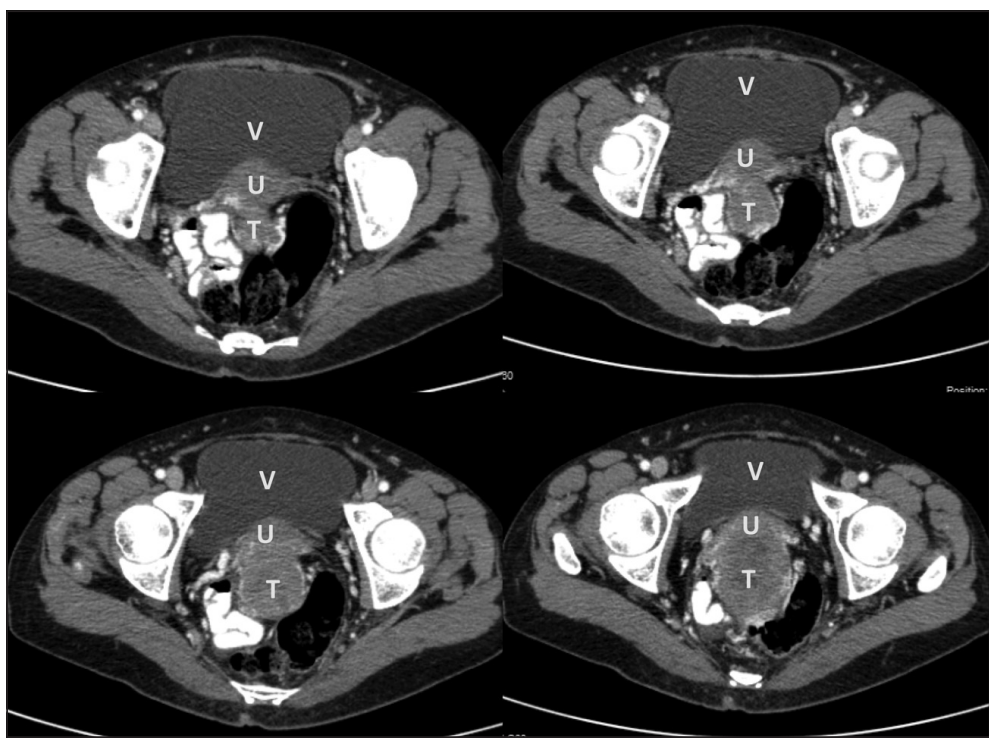

Figura 1. TC pelvis. Masa tumoral desplaza e infiltra vagina, útero y recto. $\mathrm{V}=$ vejiga; $\mathrm{U}=$ útero; $\mathrm{T}=$ tumor. 


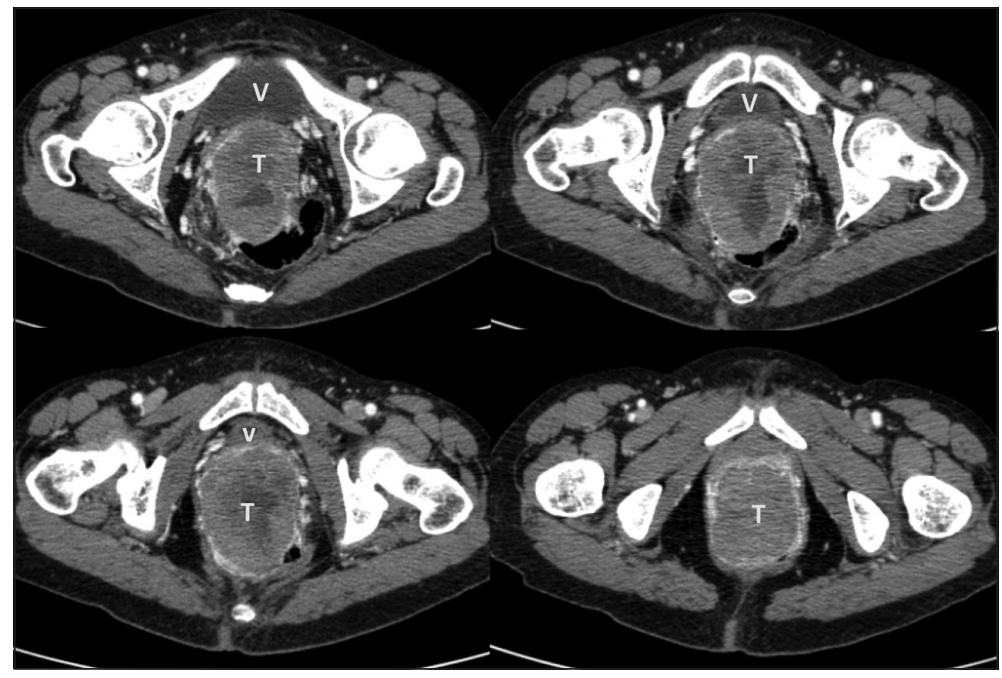

Figura 2. TC pelvis. Masa tumoral desplaza e infiltra vagina y recto.

$\mathrm{V}=$ vejiga; $\mathrm{T}=$ tumor.
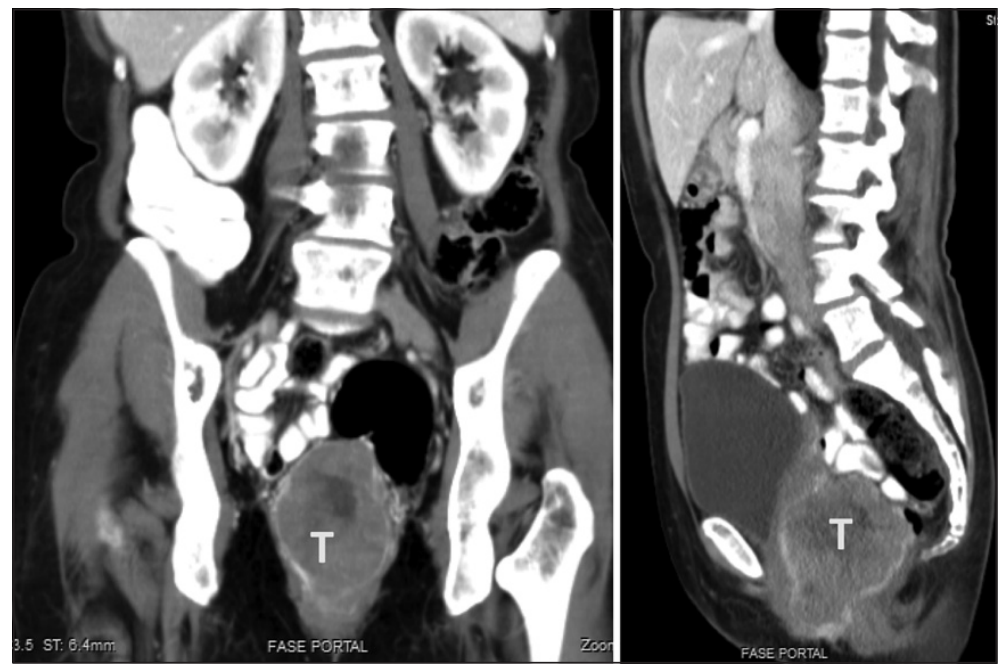

Figura 3. TC pelvis. A. Corte Coronal. B. Corte Sagital.

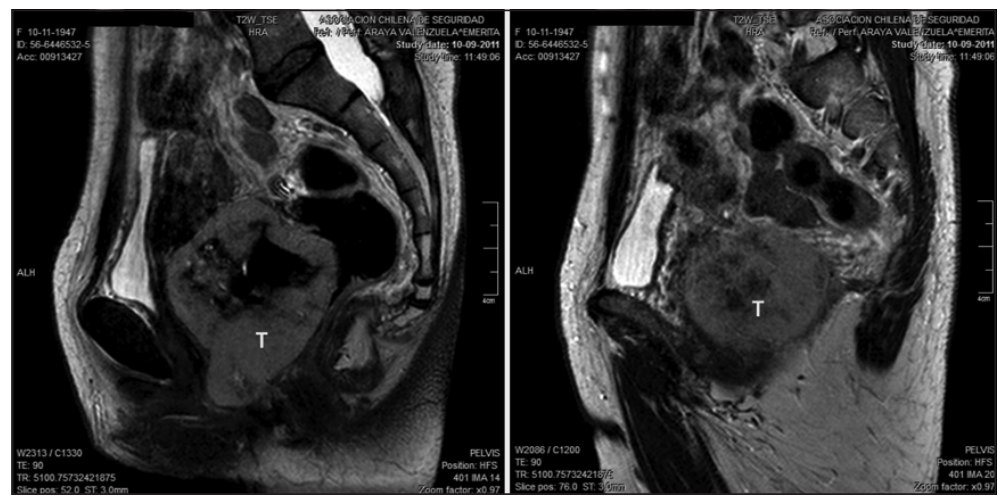

Figura 4. Tumor gigante que ocupa el fondo de saco recto-uterino. 


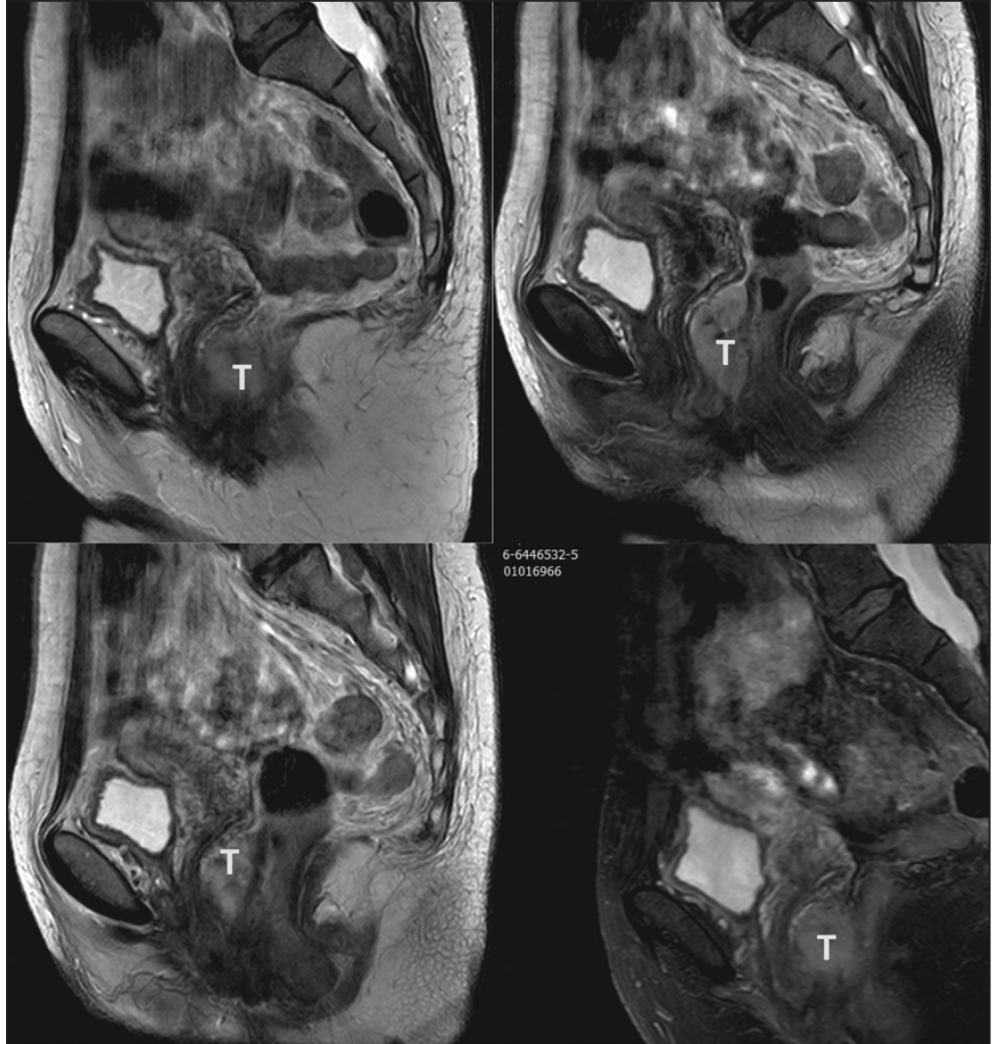

Figura 5. RM: control a los 2 meses de tratamiento.
S 100 (-) (Figura 6). Evolución postoperatoria sin incidentes. Se mantiene en control clínico cada 2 meses y luego de completar 18 meses de imatinib $400 \mathrm{mg}$, la paciente ha recuperado su peso normal, refiere continencia plena y no presenta signos de recidiva local. Presentó como efectos colaterales de la droga edema facial, derrame subconjuntival, forunculosis glútea, síndrome de las piernas inquietas (de predominio nocturno y que responde parcialmente al Nervoheel ${ }^{\mathrm{R}}$ sublingual), además de caída parcial del pelo, pestañas y cejas, síntomas actualmente en regresión.

\section{Discusión}

La introducción del imatinib, inhibidor de la tirosinquinasa ha transformado la terapia de los tumores GIST avanzados o metastásicos ${ }^{3-5}$. Actualmente, existe consenso en la literatura especializada que las indicaciones formales de neoadyuvancia con Imatinib en el manejo de los GIST de recto son: 1) Tumores irresecables; 2) Facilitar la cirugía con resecciones R0; 3 ) Evitar resecciones multiviscerales de alta morbilidad; 4) Permitir cirugía radical con conservación de esfínter y 5) Lograr la extirpación total de la lesión con las ventajas obvias de una cirugía mínimamente invasiva, lo que es particularmente relevante en este tipo de tumores que raramente dan metástasis por vía linfática. La potencia del imatinib en terapias de inducción de GIST del recto de alto riesgo se expresa en tasas de respuesta del $80 \%$ de los casos tratados, logrando habitualmente una respuesta parcial y una estabilización de la enfermedad, con algunos casos reportados de respuesta patológica completa ${ }^{5,6}$. La positiva experiencia de la neoadyuvancia con imatinib se ha multiplicado en los últimos años, determinando que actualmente sea el tratamiento estándar para los GIST de recto que requieren una cirugía radical o que está en riesgo el aparato esfinteriano ${ }^{3-10}$. En una experiencia de Japón, sobre 29 pacientes tratados en la era pre-imatinib por un GIST rectal, 19 fueron sometidos a una resección abdominoperineal $^{10}$. En una revisión reciente de 39 casos atendidos en 2 centros de referencia alemanes en un período de 9 años, se concluye que la neoadyuvancia con imatinib mejora los resultados en cuanto a bordes quirúrgicos libres de tumor, sobrevida libre de enfermedad, sobrevida global y tasas de recidiva local ${ }^{11}$. 

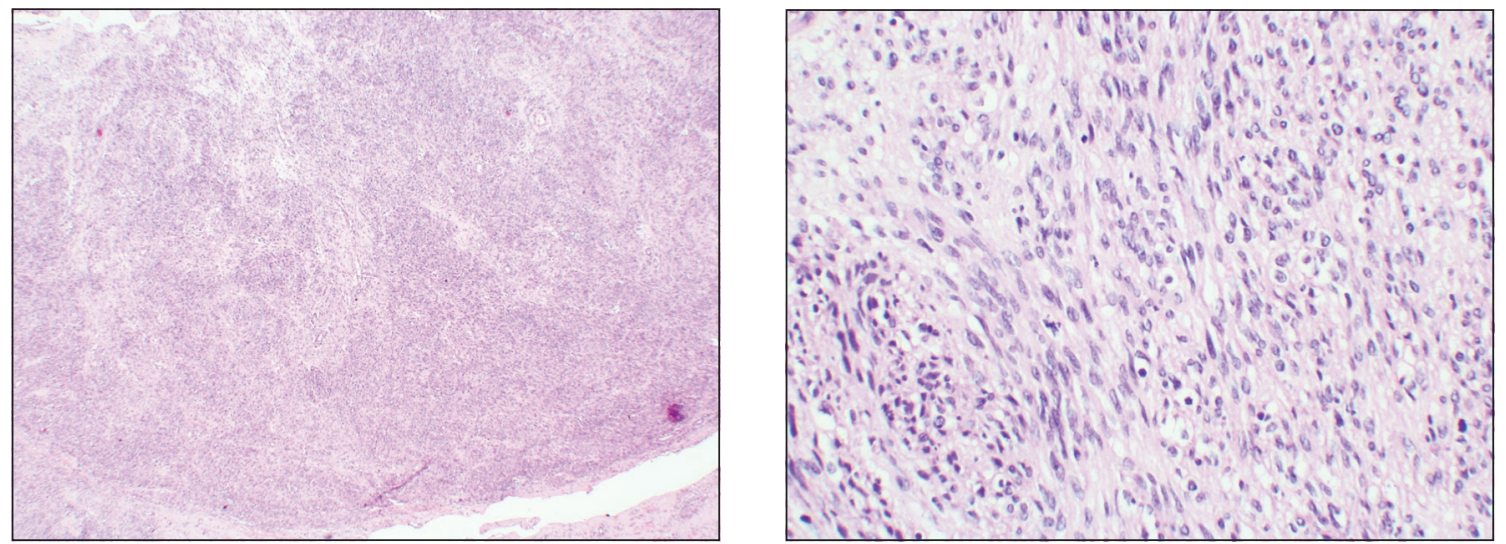

Figura 6. Histopatología. A. HE x 100: células tumorales fusadas en estroma parcialmente hailinizado. B. HE x 400: células tumorales fusadas con núcleos mitóticos.
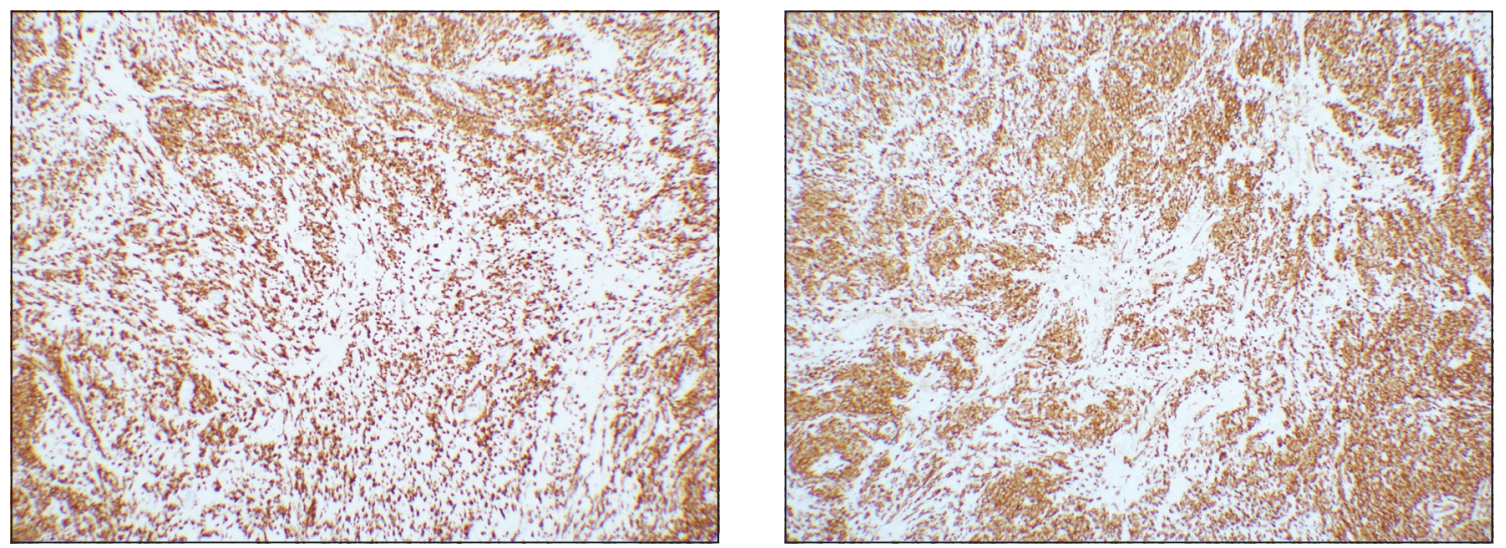

Figura 7. A. CD 117 y B. CD 34 inmunohistoquímica: positividad intensa en la mayoría de las células tumorales.

La dosis y duración de la neoadyuvancia con imatinib no está bien definida en la literatura actual, pero en general se acepta que la dosis estándar son $400 \mathrm{mg}$ que se mantienen por 3 a 6 meses, como una forma de evitar la resistencia secundaria y la progresión tumoral. Tampoco existe consenso respecto de la duración del tratamiento adyuvante con imatinib luego de la resección quirúrgica, aunque se reconoce la necesidad de mantener la terapia para reducir el riesgo de recidiva local, especialmente en los GIST malignos, es decir, tumores de más de $5 \mathrm{~cm}$ y/o con un índice mitótico alto ( $>5$ x 50 CAM). La mayoría de los autores prolonga el tratamiento por 18 a 24 meses, aunque hay reporte de pacientes tratados por 4 a 5 años. La resistencia primaria al imatinib ocurre en el $11 \%$ de los casos, lo que dependería del tipo de mutación, hecho que ha estimulado el empleo de nuevas drogas en el control de los GIST malignos ${ }^{12}$.

\section{Referencias}

1. Miettinen M, El-Rifai W, Sobin LH, Lasota J. Evaluation of malignancy and prognosis of gastrointestinal stromal tumours: a review. Hum Pathol. 2002;33:47883.

2. De Matteo RP, Lewis JJ, Leung D, Mudan SS, Woodruff JM, Brennan MF. Two hundred gastrointestinal stromal tumors: recurrence pattern and prognostic factors for survival. Ann Surg. 2000;231:51-8.

3. Yoon KJ, Kim NK, Lee KY, Min BS, Hur H, Kang J, et al. Efficacy of imatinib mesylate neoadjuvant treatment for a locally advanced rectal gastrointestinal stromal tumor. Korean Soc Coloproctol. 2011;27:147-52.

4. Hou Y-Y, Zhou Y, Lu S-H, Qi W-D, Xu C, Hou J, et al. Imatinib mesylate neoadjuvant treatment for rectal malignant gastrointestinal stromal tumor. World J Gastroenterol. 2009;15:1910-3. 
5. Grassi N, Cipolla C, Torcivia A, Mandalà S, Graceffa G, Bottino A, et al. Gastrointestinal stromal tumour of the rectum: report of a case and review of literature. World J Gastroenterol. 2008;14:1302-4.

6. Silva de Azevedo CRA, Paiva Jr TF, Rossi BM, Guimaraes GC, de Souza Begnami MDF, Oliveira TB, et al. Pathologic complete response with neoadjuvant imatinib for locally advanced pelvic GIST. Int J Clin Oncol. 2011;16:279-83.

7. Machlenkin S, Pinsk I, Tulchinsky H, Ziv Y, Sayfan J, Duek D, et al. The effect of neoadjuvant imatinib therapy on outcome and survival after rectal gastrointestinal stromal tumour. Colorectal Dis. 2011;13:1110-5.

8. Dickhoff C, Leguit RJ, Slors JFM, Vervenne WL, Bemelman WA. Giant rectal gastrointestinal stromal tumors: a report of two cases. Case Rep Gastroenterol. 2008;2:54-9.
9. Wang JP, Wang T, Huang MJ, Wang L, Kang L, Wu XJ. The role of neoadjuvant imatinib mesylate therapy in sphincter-preserving procedures for anorectal gastrointestinal stromal tumor. Am J Clin Oncol. 2011;34: 314-6.

10. Hamada M, Ozaki K, Horimi T, Tsuji A, Nasu Y, Iwata $\mathrm{J}$, et al. Recurrent rectal GIST resected successfully after preoperative chemotherapy with imatinib mesylate. Int J Clin Oncol. 2008;13:355-60.

11. Jacob J, Mussi C, Ronellenfitsch U, Waldelmann E, Negrio T, Gronchi A, et al. Gastrointestinal stromal tumor of the rectum: results of surgical multimodality in the era of imatinib. Ann Surg Oncol. 2013;20:58692.

12. Judson IR. Prognosis, imatinib dose, and benefit of sunitib in GIST: knowing the genotype. J Clin Oncol. 2008;26:5322-5. 